\title{
Two-scale mass-flux closure models for turbulence: p-mode amplitudes in solar-like stars

\author{
K. Belkacem, ${ }^{1}$ R. Samadi, ${ }^{1}$ M.-J. Goupil, ${ }^{1}$ F. Kupka, ${ }^{2}$ M.-A. Dupret ${ }^{1}$ \\ ${ }^{1}$ Observatoire de Paris, LESIA, CNRS UMR 8109, 92195 Meudon, France \\ 2 Max-Planck-Institute for Astrophysics, Karl-Schwarzschild-Str. 1, 85741 Garching, Germany
}

\begin{abstract}
A new closure model has been developed, which takes into account both the skewness of the velocity distribution induced by the presence of two flows in the convection zone, and the effects of turbulence onto each flow (Belkacem et al. 2006a). Applied to the formalism of $p$-mode excitation, it has been possible to validate this theoretical model by a comparison with the observational excitation rates in the solar case using GOLF data (see Belkacem et al. 2006b). The next step is to consider $\alpha$ Cen A for which observations of the mode-damping rates are available.
\end{abstract}

\section{Results and conclusion}

A confrontation of the solar excitation rates using the closure model with plumes (CMP) has successfully been performed in the solar case, except at high frequency where uncertainties remain. In these proceedings we focus on $\alpha$ Cen A for which the best data are available (Bedding et al. 2004, Fletcher et al. 2006). These constraints are compared with new theoretical calculations as developed by Belkacem et al. (2006b).

Figure 1 shows that the current observational constraints are not accurate enough to discriminate between the quasi-normal approximation (QNA) closure model (see Belkacem et al. 2006a) and the CMP although, as in the solar case, the CMP decreases the discrepancy between theoretical and observational excitation rates. Preliminary work tends to show that the asymmetry between the updrafts and the downdrafts does not change significantly between the few 3D simulations of main-sequence stars investigated in this work and that the CMP remains valid according to these 3D simulations. Hence, we expect that the difference between the effect on the excitation rates of the CMP model and that of the QNA remains constant for intermediately massive stars lying on the main sequence. This, however, needs to be confirmed using more 3D simulations and needs to be extended to other domains in the HR diagram. It emphasizes the need for more accurate seismic data to discriminate between the two closure models. The space based mission CoRoT is an asteroseismology mission that in the very near future will enable us to derive, for a large set of solar-like oscillating stars with different effective temperature and gravity, the rates at which energy is supplied to the modes by turbulent convection. The quality of those data is expected to be significantly higher than for current observations.

\section{References}

Bedding T. R., Kjeldsen H., Butler R. P., et al., 2004, ApJ, 614, 380

Belkacem K., Samadi R., Goupil M.-J., Kupka F., 2006a, A\&A, 460, 173

Belkacem K., Samadi R., Goupil M.-J., Kupka F., Baudin F., 2006b, A\&A, 460, 183

Fletcher S. T., Chaplin W. J., Elsworth Y., et al., 2006, MNRAS, 371, 935 


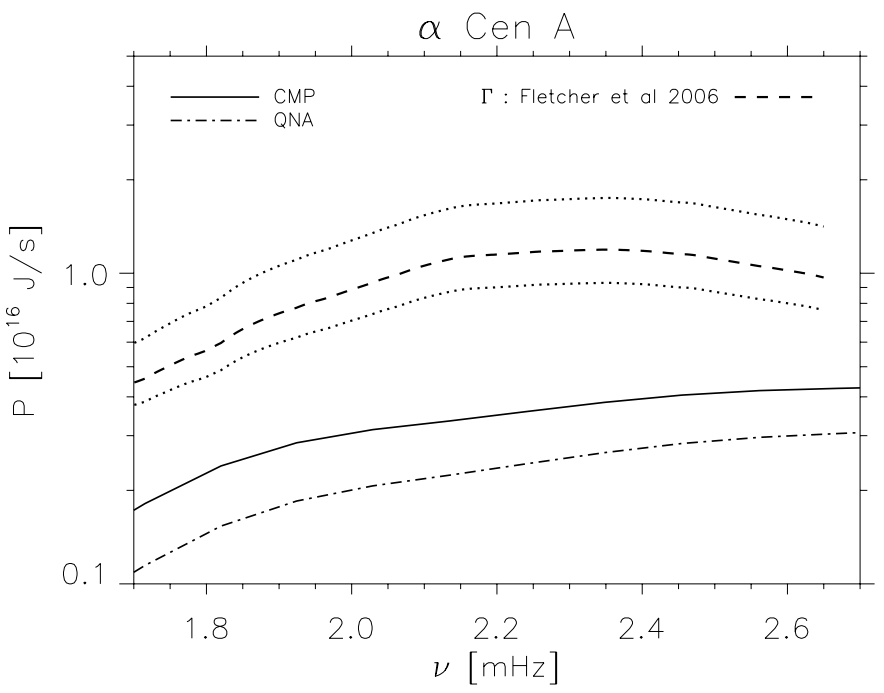

Figure 1: The dashed line corresponds to the constraints obtained from the observed spectrum derived by Bedding et al. (2004) for the amplitudes and the averaged mode line-widths derived by Fletcher et al. (2006). Dotted lines correspond to the estimated error interval of the observational data. The theoretical calculation based on the CMP is plotted as the solid line and the QNA closure based calculation as the dash-dotted line.

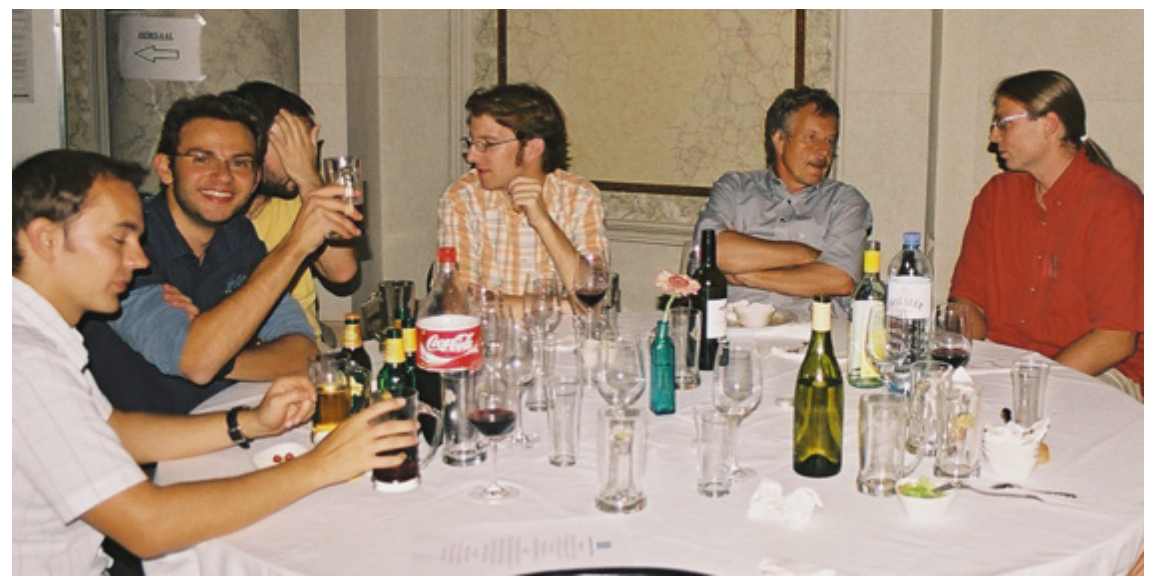

Alexander Kaiser, Paul Beck, Patrick Lenz, Gerhard Hensler and Holger Pikall. 